\title{
Exploring how students use sandbox software to move between the physical and the formal
}

\author{
Elias Euler \& Bor Gregorcic \\ Department of Physics and Astronomy, Uppsala University, Uppsala, Sweden 75120
}

\begin{abstract}
In this paper, we present a theoretical framework based on Hestenes's discussion of modeling in physics and diSessa's early theories on creativity-based digital learning environments. We use this framework to formulate new understandings of how a pair of students work with an open-ended physics sandbox software, Algodoo, alongside a physical laboratory setup. Algodoo is a digital environment that makes it possible for students to create simple, two-dimensional models of physical phenomena. We identify Algodoo's role as that of a semi-formalism, whereby the students made use of the software in their process of modeling as a means of moving between the physical, experimental context and the formal, mathematical representations associated with that context. We propose a hypothesis to be tested in future research and suggest further avenues for exploration in relation to the proposed theoretical framework.
\end{abstract}

\section{INTRODUCTION}

Recent research into the use of digital tools in physics education reflects a growing interest in investigating the potential roles of well-designed simulations [1], a class of interactive digital environments that allow students to work with constructed models of situations or phenomena [2]. Simulations are typically designed to help students better understand specific conceptual topics and, thus, are developed around a single phenomenon or collection of a few related phenomena (as, for example, with the Gravity and Orbits simulation from PhET [3] or the Inclined Plane with Two Masses and a Pulley simulation from GeoGebra [4]).

In contrast, constructionist environments, also referred to as microworlds, are designed for users to explore and create a wide range of phenomena within the same software, encouraging these users to interactively construct and deconstruct their own models [5]. In the early years of research into digitally-assisted learning in physics, diSessa [6] described a distinct role for such creativitydriven digital environments: that of a semi-formalism, which he described as providing students with access to the formal ideas of physics in ways that could be strongly related to their everyday experiences.

An example of a microworld in physics education, and a software that has not yet been researched thoroughly, is the digital sandbox software, Algodoo. In this study, we make use of diSessa's term, semi-formalism, to show how openended software like Algodoo can play a mediating role between the physical world and the formal, mathematical representations used in physics. To do so, we examine how Algodoo was used in an isolated physics activity and propose that the use of Algodoo can aid in student's mathematization by functioning as a means for modeling.

\section{MODELING \& ALGODOO}

Many education researchers see modeling (in the broad sense) to be the fundamental enterprise of physics. Notably,
Hestenes [7] claims that physics teachers should explicitly provide students with the rules by which the physics modeling "game" is played out as a scientific activity. For him, this entails that physics teachers should create learning environments that show students how modeling underpins the constitution of physics knowledge. In this way, Hestenes claims that enabling students to build, inspect, and use models is at the core of quality physics teaching.

Fig. 1 shows a modified version of Hestenes' visualization for the relationship between the physical world (bottom) and the collection of models used within the Newtonian tradition (top). In this modified version, we have included our interpretation of where a semi-formalism would reside in this system (right). DiSessa suggested that semiformalisms would provide alternative means for accessing the formal, mathematically rigorous ideas used in physics (in a manner which is more similar to their experiences of the physical world) which we show as vertical halfway point between the two worlds that Hestenes depicted. The triad of physical phenomena (Ph), semi-formalism (S-f), and formalisms (F) is also shown inset in Fig. 1.

Algodoo is a digital sandbox environment where users can draw two-dimensional shapes that fall down, bounce, swing, slide, and generally interact with one another according to Newtonian mechanics. The software is based around an open-endedness and creativity (hence, the 'sandbox' label) and, thus, allows students to construct a wide range of systems varying from the simple (e.g. spring-mass pendula) to the elaborate (e.g. suspension bridges or engine transmissions). While using Algodoo, students can create dynamic models of physical situations using geometrically simple shapes and physics-relevant objects such as springs, axels, motors, thruster engines, ropes, and gears. Within the student-created scene, the properties of each of these objects can be intentionally varied to explore how properties such as the coefficient of friction, spring constant, and even gravity/air resistance can influence the behavior of a model.

The software also includes options for displaying more mathematically formal representations such as dynamic 
vector arrows displayed on the objects, windows containing graphs, and adjustable path tracers for visualizing movement in two dimensions. Within the Algodoo software, then, students can experience both a creative, semi-formal space as well as a collection of formal representations that relate to their explorations within that space.

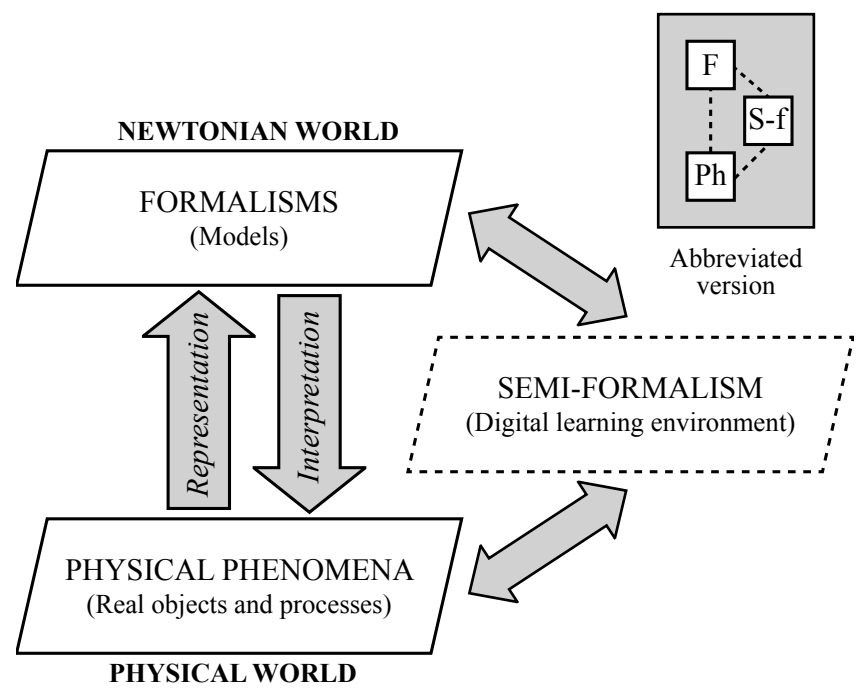

FIG. 1. Hestenes' [7] "Newtonian Epistemology" (left) modified to include a digital semi-formalism (right, shown with a dashed border), which mediates between the physical world and the formal (modeled) world.

\section{METHODS}

In this study, we analyze video recordings of a student pair as they worked with Algodoo alongside a physical laboratory setup. The participants were pre-service teachers in an introductory level physics course at a Swedish university. Pre-service teachers at this university attend a mix of physics, mathematics, and pedagogy courses during their program. The students who volunteered to participate in this study were completing their first semester of physics and mathematics at the time of data collection.

The data collection sessions began by situating the pair of students in a non-threatening work environment outfitted with Algodoo running on a large interactive whiteboard (IWB). The students were given roughly an hour to learn the basics of Algodoo and explore the functionality of the software. The pair was then presented with a physical ramp setup (consisting of a straight metal ramp, a hockey puck, and several wooden blocks for incrementing the height of one end of the ramp on a table) and orally given a task:

Using both the physical ramp and Algodoo, convince us (the researchers) of the relationship between (1) the height above the table from which the puck is released to roll down the inclined ramp and (2) the horizontal distance from the edge of the table which the puck travels before hitting the ground.
The task was designed purposefully to support the inclusion of the three domains in which we were interested.

Video of the students was recorded as they worked, which lasted approximately an hour, and during which time we (the two researchers) remained present to answer questions about the software, encourage explication from the students, and ensure that the students made use of both $\mathrm{Al}$ godoo and the physical ramp setup. The data that we analyze for this study comes from the second portion of the session where the students worked on the ramp task.

To analyze the students' actions during the session, we generated a multimodal transcription [8] of the video in which we explicitly notated the students' talk, gesture, and interaction with objects (e.g. swiping on the IWB or lifting/tilting the ramp). Based on a careful consideration of the transcripts and frequent comparisons with the original video clips, we tracked how the students came to understand the situation by moving between three domains within the activity (which, as we discuss below, correspond to the triad of the physical phenomenon, semi-formalism, and formalisms): the physical ramp, the two-dimensional scene within Algodoo (where digital objects were created and interacted), and mathematical representations of motion (in this case, $\mathrm{x}-\mathrm{y}$ position graphs generated within Algodoo). That is, we followed how students created and interpreted their two-dimensional model in Algodoo from a multimodal perspective to track how each of the domains was involved in their process of meaning making.

\section{FINDINGS}

The students involved in the study were ultimately able to mathematically relate the two desired variables (height of one end of the ramp above the table and distance along the floor which the puck traversed) through their use of both the physical setup and a digital model within Algodoo. This constructed digital model was made up of an angled rectangle corresponding to the ramp, a horizontal rectangle corresponding to the portion of the table extending after the ramp, and a circle which was allowed to roll down and across the two rectangles before landing on the 'ground' (an infinite plane automatically generated in Algodoo). The students began the activity by creating the digital model to check their intuitions about the physical situation, focusing on the fact that there's a point where tipping the ramp more results in the puck travelling a shorter distance along the floor as it bounces more at the transition to the table. The students then went on to collect several data points with the physical set up, which they plotted on a graph and then used to interpret the functional shape for smaller angles before bouncing off the table caused diminishing returns.

As examples of our use of multimodal analysis, we focus now on three exchanges that occurred during the students' construction of the digital model to highlight instances where the students moved between the three domains to make meaning (Fig. 2). Each illustration is 


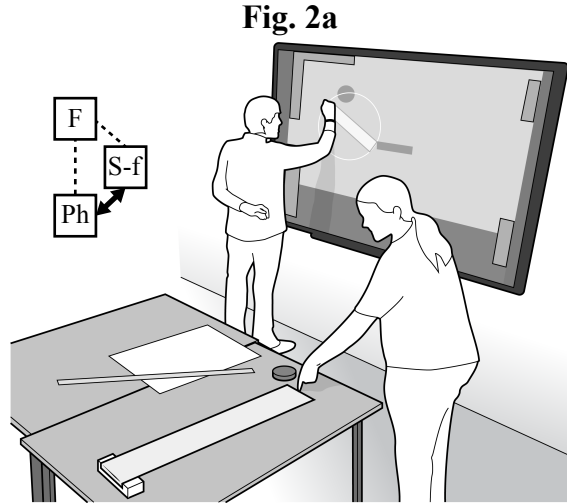

S2: Is there any way for this software to change the center of rotation?

R: Where would you like the center of rotation to be then?

S2: Down here. [points to the base of the ramp]
Fig. 2b

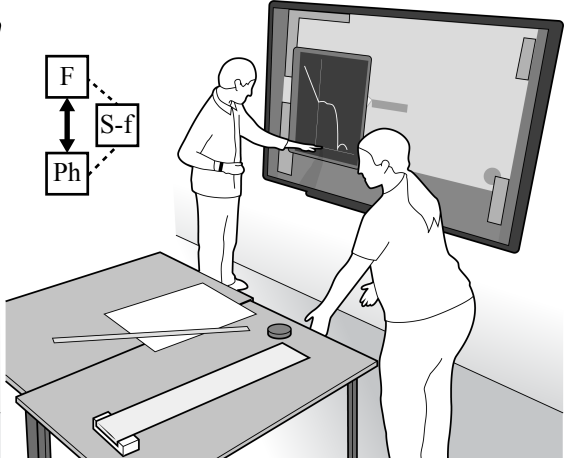

S2: We have to look at it from here to there. [points to the graph]

S1: Hits the ground there. [points to the graph] That's what we want.

S2: Yeah we want to know the distance... here? [gestures to show horizontal distance from table]
Fig. 2c

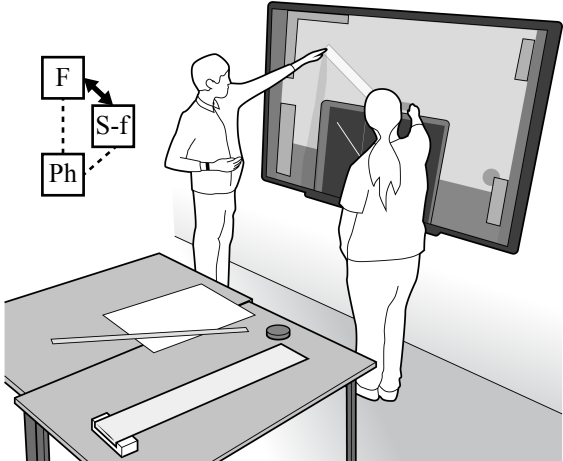

R: And where would you like [the zero] to be?

\section{S1: Here. [points to the top of the tilted rectangle]}

S2: No... we want it on the end there. [points to the right edge of the horizontal rectangle]

FIG. 2. Illustrations (from this source video [9]) of student activity (with captions and Fig. 1 insets). The S1 label refers to the student in the center of the image, S2 refers to the student on the right, and R refers to one of us, the researchers present during the activity. 2a: An example where $\mathrm{S} 2$ used the bottom of the physical ramp to explain the position around which he wanted a rectangle in Algodoo to rotate (showcasing movement between the physical domain $(\mathrm{Ph})$ and the semi-formal domain (S-f)). 2b: An example where S2 used an environmentally-coupled gesture to clarify one of the relevant distances for the prompt while trying to interpret a plot of the moving circle's position in Algodoo (showcasing movement between the physical domain $(\mathrm{Ph})$ and the formal domain $(\mathrm{F})$ ). 2c: An example where both $\mathrm{S} 1$ and S2 point to locations within the Algodoo scene to demonstrate where they wanted to align the $\mathrm{y}$-axis $(\mathrm{x}=0)$ in a graph they had generated (showcasing movement between the semi-formal domain (S-f) and the formal domain (F)).

accompanied by the transcribed talk that occurred during the chosen exchanges and a diagram to illustrate which of the three domains were utilized by the students for making meaning. In each of these examples, the students were not explicitly directed by the researchers to incorporate multiple domains (or any specific domain, for that matter).

Fig. 2a shows an exchange during the first steps of the digital model construction. The students had set up the objects in the model and were trying to determine how to rotate the tilted rectangle around its corner rather than its center (the center being the default for rotations in Algodoo). One student then asked if there was a way to achieve this rotation in Algodoo. When one of us responded by asking where the student desired the center of rotation to be, they spontaneously replied by pointing to the end of the physical ramp instead of the corner of the rectangle within Algodoo. This example showcases a student using the physical domain to elaborate a point within the semi-formal domain.

Fig. $2 b$ shows an exchange after the students had created the digital model and were trying to track the horizontal distance that the circle travelled before hitting the ground within Algodoo. To do this, the students generated a plot of the y-position vs. the x-position of the circle. However, as the students interpreted their plot, they wanted to move the location of the $y$-axis $(x=0)$ so they could read off the $x$ - value directly as the horizontal distance. To clarify which distance they were attempting to measure, one student gestured next to the physical table while he posed a question to the researchers. This exchange showcases an example of a student using the physical domain to make meaning in the formal domain of the graph (note that, in our examples, both the formal domain and the semi-formal domain are accessed through Algodoo; it is not the presence of the software or the IWB that determines the domain but rather the manner in which the software and IWB are used). We treat the graph within Algodoo as part of the formal domain because it conveys information through mathematical resources that physicists use to model phenomena (specifically, a coordinate system being used to plot a graph).

Fig. 2c shows an exchange where the students were deciding where to place the $y$-axis of their graph so that they could read off the horizontal distance as the $\mathrm{x}$-value without an offset. As the students determined how to move the axis of the graph, we asked them where they would like the axis to be within the plot. The students both pointed to positions on the rectangles in the digital model where they thought the ideal position for the y-axis of their graph would be. This exchange showcases an example of the students using the semi-formal domain to explain their reasoning about an aspect of the formal domain. 


\section{DISCUSSION}

Our findings show how the students related semiotic information across the three domains (physical, formal and semi-formal) to make meaning with their talk, gesture, and interaction with the environment. Through their domaindistributed meaning making, the students made use of all three of these domains, and frequently moved between them to construct a mathematized version of the physical phenomena of a puck rolling down a ramp, ultimately determining how the height of the ramp can be mathematically related to distance the puck travels off of the edge of the table.

While we do not claim that these students were only able to arrive at their mathematical conclusions due to the inclusion Algodoo, we do assert that the students' use of the digital environment was a key factor in their developing an understanding of the phenomenon and the mathematically formal physics associated with it. Despite the domains having different dimensionality and varied levels of mathematical abstraction, the students frequently moved between the three domains with ease as they constructed and utilized their digital model of the physical ramp.

It should be pointed out that, during the activity, we did not include any explicit discussion of the rules of the modeling "game" that the students played, contrary to Hestenes' [7] recommendations. Nonetheless, with a better theoretical conception of the role that creativity-driven digital environments might play in the modeling processes of physics students, we suggest that software like Algodoo could be a useful tool for teachers to discuss modeling in the explicit manner that Hestenes suggests and even as an object for discussing the epistemological issues surrounding the use of computer-generated models in the practice of science [10].

Our findings are presented from a single, exploratory case which should not be generalized to predict how every student will utilize open-ended digital learning environments. We can, however, use this case to propose the following hypothesis to be tested in the future:

The inclusion of creativity-based digital learning environments (like Algodoo) in physics activities increases the likelihood that students will draw conceptual parallels between

[1] W. K. Adams, S. Reid, R. LeMaster, S. B. McKagan, K. K. Perkins, M. Dubson, and C. E. Wieman, J. Interact. Learn. Res. 19, 397 (2008).

[2] J. L. Plass and R. N. Schwartz, in Cambridge Handb. Multimed. Learn., edited by R. E. Mayer (Cambridge University Press, Cambirdge, UK, 2014), pp. 729-761.

[3] https://phet.colorado.edu/en/simulation/gravity-andorbits, (retrieved Jul 1, 2017).

[4] https://geogebra.org/material/show/id/57587 (retrieved Jul 1, 2017).

[5] S. Papert, Mindstorms: Children, Computers and Powerful Ideas (Basic Books, New York, NY, 1980). the physical world and the formalisms used in physics.

This hypothesis, whose exploration would surely aid in understanding the degree to which digital environments can be leveraged as semi-formalisms, might be tested in further empirical studies by observing how students recruit and interpret mathematical formalisms during physics activities when Algodoo is included as compared to students who use other more typical (single-phenomenon) simulation software and/or no digital environment at all.

We also intend to further explore the relationship between the three domains proposed in this paper from different theoretical perspectives. For example, the triad of domains might be seen as being characterized by their degree of messiness. One of major critiques of simulation use in physics learning (especially as substitutes for laboratory work) is that students are presented with all of the important and relevant features of a phenomenon and they never encounter the messiness of physical laboratory work $[11,12]$. We suggest here that the open-endedness of creativity-driven digital learning environments like Algodoo allows for more authentic, messier inquiry than traditional simulations. Appropriate use of Algodoo might entail a higher degree of messiness by requiring students make modeling and representational decisions and by allowing them the freedom to explore and investigate their own models' behavior through deconstruction. Still, as compared to the physical world, we suggest that Algodoo provides more direct (less messy) access to some formal representations, such as graphs, without the need for students to perform the process of measurement (which, in itself, might be seen as a messy procedure).

In this paper, we have explored the potential for openended modeling environments such as Algodoo to enhance the teaching and learning of physics. Nonetheless, there exists more to be investigated as to the educational potential of open-ended digital learning environments, especially as a means for students to actively participate in modeling.

\section{ACKNOWLEDGEMENTS}

This project is funded in part through the Swedish Research Council grant 2016-0411.

[6] A. DiSessa, in Constr. Comput. Age, edited by G. E. Forman and P. B. Pufall (Lawrence Erlbaum, Hillsdale, NJ, 1988), pp. 49-70.

[7] D. Hestenes, Am. J. Phys. 60, 732 (1992).

[8] A. Baldry and P. T. Thibault, Multimodal Transcription and Text Analysis (Equinox, London/Oakville, 2006).

[9] https://youtu.be/q5hAYy-dpPU, (retrieved Jul 1, 2017).

[10] I. M. Greca, E. Seoane, and I. Arriassecq, Sci. \& Educ. 23, 897 (2014).

[11] J. Bryan, Contemp. Issues Technol. Teach. Educ. 6, 230 (2006).

[12] C. Chinn and B. Malhotra, Sci. Educ. 86, 175 (2002). 